in experimental social psychology. Vol. 6, New York: Acad emic Press, 1972.

Schlosberg, H. Three dimensions of emotion. Psychological Review, 1954, 61, 81-88.

Wing, H., \& Nelson, C. E. The perception of personality through trait sorting: A comparison of trait sampling techniques. Multivariate Behaviral Research, 1972, 7, 269-274.

Woodworth, R. S. Experimental psychology. New York: Holt, 1938.
NOTE

1. In the study of personality traits no difference was found between using each trait in only one description and using a trait in as many descriptions as the $S$ desired (Wing \& Nelson, 1972).

\title{
Effects of thirst drive on cue utilization and cue dominance of spatially separate cues in albino rats*
}

\author{
JEROME S. COHEN and GISELLE TUBARO \\ University of Windsor, Windsor, Ontario, Canada N9B 3P4
}

\begin{abstract}
Albino rats under moderate $(18 \mathrm{~h} /$ day $)$ or high $(23.5 \mathrm{~h} /$ day $)$ water deprivation we re required to learn either a central door cue or peripheral wall cue simultaneous discrimination in a two-choice discrimination apparatus. Following this acquisition phase, a series of redundant-relevant cue trials with both door and wall cues were introduced. Animals were tested on the utilization of wall and door cues separately and on the dominance for each cue. Drive level only affected original discrimination acquisition of the peripheral wall-cue task. Moderate-drive animals were better able to learn the wall-cue discrimination than high-drive animals. In general, door-cue learners were able to utilize both cues but showed dominance for door over wall cues. Wall-cue learners, however, were only able to utilize wall cues and showed dominance for wall over door cues.
\end{abstract}

Drive level has been shown to be inversely related to exploratory activity of rats in reinforced complex mazes (Chapman \& Levy, 1957; Cohen \& Stettner, 1968; Hughes, 1965) and to be inversely related to cue utilization of redundant-relevant cues in discrimination tasks (Bruner, Matter, \& Papanek, 1955; Cohen, Stettner, \& Michael, 1968; Telegdy \& Cohen, 1971). In view of the similar relationships between drive and these two separate behaviors, cue utilization could be considered to be mediated by exploratory activity. Increased exploratory activity under decreased drive level may increase the likelihood that animals would sample more redundant-relevant stimuli. This possibility is suggested by recent findings (Cohen \& Sullivan, 1973) in which only moderately water deprived $(18 \mathrm{~h})$ rats were disrupted in their responses to learned central brightness cues by changes in location of redundant-relevant peripheral cues. The present study is an extension of the recent Cohen and Sullivan research (1973). As such, it represents an indirect test of the proposed exploration mediation hypothesis of cue utilization. Animals under high- $(23.5 \mathrm{~h})$ and moderate-water $(18 \mathrm{~h})$ deprivation were required to learn a brightness discrimination from either central door or peripheral wall brightness cues.

*The present study was conducted under a National Research Council of Canada Grant (APA 7450).
Redundant-relevant peripheral or central cues were then added to the learning environment. Animals were then tested for their utilization of each cue separately and for their cue preference, i.e., cue dominance, in an opposed cues test. According to the exploration mediation hypothesis, original discrimination acquisition of the peripheral wall cues should be more difficult for the high- than the moderate-drive groups. Furthermore, it was expected that only moderately deprived rats would be able to utilize their initially learned and the incidentally placed redundant-relevant cues. Highly deprived animals were expected to be able to utilize only their originally acquired cue. The design of the experiment also allowed for the investigation of drive level effects on cue dominance.

\section{METHOD}

\section{Subjects}

Thirty-six male albino rats (Wistar strain) approximately 80 days old at time of training and shaping were purchased from Woodlyn Farms, Guelph, Ontario. Two animals failed to run in the experimental sessions and were replaced by substitute rats from the same breeding stock.

\footnotetext{
Apparatus

A two-choice discrimination box, as previously described (Telegdy \& Cohen, 1971), was employed. Two $10 \times 10 \mathrm{~cm}$ stimulus doors led directly into goal chambers. The side walls formed a V-shaped decision chamber. Each radiating wall
} 
contained a removeable $10-\mathrm{cm}$ high panel. During pretraining or shaping, the interior of the appartus was flat gray. During discrimination training and the test phase, one of the doors and/or one of the side wall panels contained vertical 2 -cm black stripes 1.3-cm apart.

\section{Procedure}

The basic pretraining and training procedures were similar to those used in the previous study (Cohen \& Telegdy, 1970). During pretraining, animals were trained to open gray stimulus doors for a 10 -sec drink of $10 \%$ sucrose solution. By the end of this phase, each animal received 12 spaced trials per day. Batches of 8-10 animals were run at a time so that intertrial intervals ranged from 4 to $6 \mathrm{~min}$. On the last pretraining day, one of the doors was locked, and an E-corrected procedure was employed, i.e., the animal was immediately placed into the start chamber again after approaching the locked door and allowed to rerun the trial. This procedure was carried out unt il the animal opened the unlocked door. Position of the unlocked door between trials was determined by a Fellows's (1967) random sequence. These procedures were maintained throughout the remaining portion of the experiment. During the pretraining period all animals were maintained on a $21-\mathrm{h}$ water-deprivation schedule.

Following the pretaining phase, animals were randomly and equally divided into high and moderate water deprivation groups. Animals maintained under high-deprivation conditions were deprived of water for $23.5 \mathrm{~h} / \mathrm{day}$ and received 0.5 - h water in their home cage after every daily running session. Animals maintained on the moderate-deprivation schedule were deprived of water for $18 \mathrm{~h}$ and received 6-h water in the same manner. Previous studies had also used these deprivation schedules (Cohen et al, 1969; Cohen \& Telegdy, 1970; Telegdy \& Cohen, 1971). In those studies, these levels resulted in differentiated discrimination learning while still maintaining reinforced instrumental responding. Prior to discrimination training, each rat was allowed to become accustomed to its respective deprivation schedule for 3 days.

Within each drive group, animals were randomly and equally divided into door and wall discrimination groups so that each group had nine rats. For the door discrimination, the positive stimulus was the striped door. Both walls were gray. For the wall discrimination, the positive stimulus was the striped wall panel, both doors being gray. During discrimination training, only the door containing the positive stimulus, for the door discriminators, or the door on the same side as the striped wall panel, for the wall discriminators, was unlocked. Each rat received 12 spaced E-corrected trials per day until it made no more than two errors within two trial blocks (24 trials) or had run for 20 trial blocks, whichever came first. In this and following phases, position of stimuli was determined by Fellows's (1967) random sequence.

After reaching discrimination criterion or 20 trial blocks, the animal received two blocks of redundant-relevant cue trials. On these trials, the animal was presented with a striped wall and door in the same position on each trial. Following these two sessions, cue-utilization and cue-dominance test trials were introduced within each session over the next 12 days. Every fourth trial was a test trial while the nine intertest trials were redundant-relevant cue discrimination trials. On each test day the animal received a door test, wall test, and opposed cues test trials. During a test trial both doors were unlocked and choice of either door led to reinforcement. The interspersion of free choice test trials was a procedure recommended by Sutherland and Holgate (1966) to prevent such trials from becoming specific learning trials.

The door-cue test trials assessed the animal's ability to utilize central cues when peripheral wall cues were held constant. Both walls had striped panels while only one of the doors contained the positive stimulus. Choice of either door was reinforced, but selection of the striped door was considered the correct response within the central-cue dimension.

The wall-cue test trials assessed the animal's ability to utilize peripheral cues when central cues were held constant. Both doors were striped, while walls were differentiated by the striped cue. Choice of either door was reinforced, but selection of the door on the side of the striped wall was a correct response within the peripheral-cue dimension.

The opposed-cues test trials determined the dominance of central or peripheral cues in controlling the animal's approach behavior. Differential wall and door cues were presented on opposite positions. Choice of either door was reinforced, but selection of the striped door was considered a central-cue choice. Selection of the door at the striped wall position was a peripheral-cue choice. The temporal order of the three test rials were randomized over the twelve test sessions.

In the present study, the floor of the apparatus was wiped with a damp cloth between trials and sessions to prevent the accumulation of redundant visual and olfactory cues.

\section{RESULTS}

During the original discrimination task, animals on the door task required fewer errors to criterion (mean errors: high-deprivation group $=32$, moderate-deprivation group $=39$ ) than either deprivation wall discrimination group (high $=102$; moderate $=76$ ). A two-way analysis of variance showed a significant task effect $[F(1,32)=76.60, p<.01]$ and a significant Task by Drive interaction $[\mathrm{F}(1,32)=7.29$, $\mathrm{p}<.05$ ]. Individual comparisons (Newman Keuls) determined the source of this interaction. Drive level did not significantly differentiate animals on the door-cue task but did so for animals on the wall-cue task. In the latter task, high-drive animals made significantly more errors than moderate animals $(\mathrm{p}<.01)$. Within each drive level, animals on the wall-cue discrimination made significantly more errors than animals on the door-cue task $(\mathrm{p}<.01)$. It should also be noted that, whereas all 18 door-cue task animals acquired their discrimination with 20 trial blocks, only 6 out of the 18 wall-cue animals were able to reach acquisition criterion. These task group differences were significant $\left(X^{2}=9.37\right.$, $\mathrm{df}=1, \mathrm{p}<.01)$. A drive level difference was also observed in that only one out of nine high-drive animals was able to learn the wall-cue discrimination, a significant proportion $(p=.02$, binominal test). In the moderate-drive group, five out of nine learned the wall-cue task, a nonsignificant proportion. Although the one high-drive learner did not make errors (35) outside of the range made by high-drive door-cue learners, moderate-drive animals that learned the wall-cue discrimination made significantly more errors $(\overline{\mathrm{X}}=65)$ than moderate-drive animals on the door-cue discrimination $(p \leqslant .05)$, Mann-Whitney $U$ test $)$. These results further indicate that peripheral wall-cues were less salient than central door-cues within the brightness dimension.

Individual $\mathrm{t}$ tests within ach test and between the door-cue and wall-cue tests confirmed observations on 
Table 1

Mean Door-Cue and Wall-Cue Responses on Wall-, Door-, and Opposed-Cues* Test Trials for Original Discrimination Learners

\begin{tabular}{llllcc}
\hline $\begin{array}{c}\text { Original } \\
\text { Discrimination }\end{array}$ & Drive & $\mathrm{N}$ & $\begin{array}{c}\text { Wall } \\
\text { Cue }\end{array}$ & $\begin{array}{c}\text { Test } \\
\text { Door } \\
\text { Cue }\end{array}$ & $\begin{array}{c}\text { Opposed } \\
\text { Cue }\end{array}$ \\
\hline \multirow{2}{*}{ Door Cue } & High & 9 & 8.7 & 11.9 & 12.0 \\
& Moderate & 9 & 8.7 & 11.4 & 11.1 \\
\multirow{2}{*}{ Wall Cue } & High & 1 & 8 & 10 & 11 \\
& Moderate & 5 & 8.2 & 6.8 & 9.4 \\
\hline
\end{tabular}

Note-Actual scores in italics for the one high wall-cue learner. *On the opposed-cues test trials, scores refer to door-cue choices for animals on the original door-cue discrimination and wall-cue choices for animals on the original wall-cue discrimination.

animals that acquired their initial discrimination (see Table 1). That is, within each drive level, animals that initially learned the door-cue discrimination showed door- and wall-cue utilization by significantly choosing those cues above chance (6 out of 12 times) on the separate cues test trials $(p<.01)$. The dominance of the door cue for these animals was also seen in their significantly greater door-cue choices above chance (6 out of 12 choices) in the opposed-cues test $(p<.01)$ and in their significantly fewer "errors" on door-cue than wall-cue tests $(p<.01)$. Only for the five moderate-drive animals that had learned the wall-cue discrimination could the same statistical analyses be carried out. These moderate-drive animals showed significant wall-cue utilization $(p<.05)$ but failed to display significant door-cue utilization. Significant wall-cue dominance was seen on the opposed-cues trials and by the significantly greater wall- than door-cue responses on the separate-cues trials $(p<.05)$. The high-drive wall-discrimination learner appeared to be able to utilize both wall and door cues and also showed great wall-cue dominance on the opposed-cues tests. No significant cue utilization or dominance differences between drive levels for animals on the door-cue task were found (Mann-Whitney Utests). Door-cue discrimination learners in each drive group made significantly more "correct" door-cue test choices than moderate-drive wall-cue learners ( $\mathrm{p}<.01$, Mann-Whitney U tests).

Similar statistical tests were carried out on animals that failed to reach acquisition criterion on the wall-cue discrimination. For the moderate-drive animals $(n=4)$, mean correct choices on the wall- and door-cue tests were 8.5 and 7.7 , respectively. This group also made 7.2 mean wall choices on the opposed-cues test. Only the results from the wall-cue test were significantly above chance level $(p<.025)$ to indicate any wall-cue utilization. For the high-drive rats $(n=8)$ mean correct choices on the wall- and door-cue tests were 7.2 and 8.5, respectively. The high-drive group only displayed signficant door-cue utilization $(\mathrm{p}<.025)$. Their door choices on the opposed-cues test $(X=5.5)$ did not differ significantly from chance. Therefore, the wall-cue nonlearners appeared to have gained some information about the discrimination. The moderate-drive animals seemed to pick up the relevance of the wall cues from their initial discrimination trials while the high-drive animals picked up the door-cue relevance from the redundant-relevant cue discrimination.

\section{CONCLUSIONS AND DISCUSSION}

Findings in the present study indicate that drive level primarily affected initial discrimination learning rather than cue utilization and cue dominance. Type of initial discrimination, however, accounted for the type of cue utilization and dominance. Although acquisition of the more salient central door cues was not affected by drive level, less salient peripheral wall cues were more difficult to learn by animals under highthan by animals under moderate-thirst drive. These results confirm predictions based on the studies which show that lower deprived rats explore peripheral stimuli more than higher deprived animals, as discussed earlier. Even those moderate-deprived rats that failed to reach criterion on the initial wall-cue task, showed wall-cue utilization, while similar high-drive rats could only attend to the central door cues.

Contrary to expectations, no drive level differences in cue utilization or cue dominance were found. Door-cue learners in both drive groups were able to utilize both spatially distinct but redundant-relevant cues. Cue utilization and cue dominance were directly related to initial single cue discrimination. Door-cue learners showed greater door-cue than wall-cue utilization, and also displayed door-cue dominance. Wall-cue learners, showed greater wall-cue than door-cue utilization and also displayed wall-cue dominance. Except for the one high-drive wall-cue learner, these animals were generally unable to utilize door cues. These results have important implications for learning theory. Our findings strongly support the notion of acquired cue utilization and dominance as discussed by Sutherland and Mackintosh (1971). The notion of inherent cue dominance as the only necessary mechanism for cue selection in infrahuman organisms (Kendler, 1971) is not supported by our findings.

\section{REFERENCES}

Bruner, J. S., Matter J., \& Papanek, M. J. Breadth of learning as a function of drive level and mechanization. Psychological Review, 1955, 62, 1-10.

Chapman, R. M., \& Levy, N. Hunger drive and reinforcing ef fect of novel stimuli. Journal of Comparative \& Physiological Psychology, 1957, 50, 233-238.

Cohen, J. S., \& Stettner, L. J. Effect of deprivation level on exploratory behavior in the albino rat. Journal of Comparative \& Physiological Psychology, 1968, 66, 514-517.

Cohen, J. S., Stettner, L. J., \& Michael, D. J. Effect of deprivation level on span of attention in a multi-dimension discrimination task. Psychonomic Science, 1969, 15, 31-32.

Cohen, J. S., \& Sullivan, B. Effects of drive level on cue utilization of spatially separated redundant-relevant cues Bulletin of the Psychonomic Society, 1973, 1, 455-457.

Cohen, J. S., \& Telegdy, G. A. Effect of drive level on habit strength in a discrimination task. Psychonomic Science, 1970, 19, 27-29.

Fellows, B. Chance stimulus sequences for discrimination tasks. Psychological Bulletin, 1967, 67, 87-92.

Hughes, R. N. Food deprivation and locomotor exploration in the white rat. Animal Behavior, 1965, 13, 30-32.

Kendler, T. Continuity theory and cue-dominance. In $H . H$. Kendler \& J. T. Spence (Eds.), Essays in neo-behaviorism. New York: Appleton-Century-Crofts, 1971, Pp. 237-264.

Sutherland, N. S., \& Holgate, V. Two cue discrimination learning in rats. Journal of Comparative \& Physiological Psychology, 1966, 61, 198-207.

Sutherland, N. S., \& Mackintosh, N. J. Mechanisms of animal learning. London: Academic Press, 1971.

Telegdy, G. A., \& Cohen, J. S. Cue utilization and drive level in albino rats. Journal of Comparative \& Physiological Psychology, 1971, 75, 248-253.

(R eceived for publication July 17, 1974.) 